\title{
Empathic concern: Distinguishing between tenderness and sympathy
}

\author{
Lidewij W. Niezink • Frans W. Siero • \\ Pieternel Dijkstra $\cdot$ Abraham P. Buunk • \\ Dick P. H. Barelds
}

Published online: 15 January 2012

(C) The Author(s) 2012. This article is published with open access at Springerlink.com

\begin{abstract}
The present research proposes that empathic concern, as assessed by six items of the ERQ, consists of two separate emotions, i.e., tenderness and sympathy. To test this assumption, nine studies were conducted among, in total, 1,273 participants. In these studies participants were presented with a hypothetical scenario of someone in need, after which empathic concern was assessed. Factor analyses showed that, indeed, the ERQ items that assess empathic concern can be split up in two factors, that is, one reflecting sympathy and one reflecting tenderness. In addition, in line with previous studies, our research showed that, in response to a need-situation that reflects current needs, individuals scored higher on the ERQ factor reflecting sympathy than on the ERQ factor reflecting tenderness. Findings are discussed in terms of the practical and theoretical implications of distinguishing between sympathy and tenderness.
\end{abstract}

Keywords Empathic concern · Sympathy · Tenderness

\section{Introduction}

Empathic concern can be defined as an emotional response of compassion and concern caused by witnessing someone else

L. W. Niezink · F. W. Siero · P. Dijkstra ( $₫)$

Department of Social Psychology, University of Groningen,

Grote Kruisstraat 2/1, 9712 TS Groningen, The Netherlands

e-mail: pieterneldijkstra@ziggo.nl

A. P. Buunk

University of Groningen and Royal Netherlands Academy

of Arts and Sciences, Amsterdam, The Netherlands

D. P. H. Barelds

Department of Organisational Psychology, University

of Groningen, Grote Kruisstraat 2/1, 9712 TS Groningen,

The Netherlands in need (e.g., Stocks et al. 2011; Woltin et al. 2011). Empathic concern is believed to elicit an approach orientation toward others in need and to facilitate pro-social behavior (e.g., Batson 1991; Stocks et al. 2009). It has been suggested that empathic concern reflects the deeply rooted drive of parental nurturance as it evolved in humans to help offspring survive. Were humans not interested in protecting, helping and nurturing their vulnerable young, our species would have quickly died out. Both McDougall (1908) and Lishner et al. (2011) argue that, through cognitive generalization based on learning and experience, empathic concern is often also felt for adults and animals in need.

To assess emotional responses to individuals in needsituations, among which empathic concern, Coke et al. (1978) developed the Emotional Response Questionnaire (ERQ) that consists of 23 adjectives individuals may experience when someone else is suffering. After almost a decade of research, and on the basis of factor analyses conducted in six studies (Batson et al. 1979; Batson et al. 1983; Coke 1980; Coke et al. 1978; Fultz 1982; Toi and Batson 1982), Batson et al. (1987) concluded that, of the 23 items of the ERQ, six adjectives measure empathic concern, that is, sympathetic, moved, compassionate, tender, warm and softhearted. We would like to propose, however, that these adjectives do not assess one single emotion (i.e., empathic concern), but two: sympathy and tenderness.

Tenderness and sympathy

Our study builds on previous studies that showed sympathy and tenderness to be different aspects of empathic concern. For instance, in an experimental study, Lishner et al. (2011; see also Lishner 2003) found sympathy and tenderness to be evoked by different needs. More specifically, these authors found feelings of sympathy to be evoked when a 
human or animal was hurt or suffering, and was experiencing a current need, that is, a discrepancy on one or more dimensions of well-being. In contrast, feelings of tenderness were evoked when a human or animal was perceived to be vulnerable, even when it had no current needs. Individuals may, for instance, experience feelings of tenderness when looking in the large eyes of a child or puppy. Although the child or puppy may not have current needs, this might change in the future (see also Kalawski 2010). A young puppy may, for instance, need protection when confronted with a vicious older dog, or assistance when trying to drink from its milk bottle. Other evidence for the distinction between sympathy and tenderness was found by Shaver et al. (1987). These authors showed that lay people tend to categorize tenderness apart from sympathy: whereas sympathy falls into a 'sadness' category, tenderness falls into a 'love' category. The present research aims to show that the important distinction between sympathy and tenderness, such as made by Lishner et al. (2011), is reflected in the structure of the ERQ, an undertaking that has not yet been conducted before.

\section{The present research}

To show that the six items of the ERQ that, according to Batson et al. (1987), assess empathic concern can indeed be split up in two factors (i.e., one reflecting sympathy and one reflecting tenderness), nine studies were conducted. First, by means of factor analyses we aim to show that empathic concern can be best split up into a factor reflecting sympathy and a factor reflecting tenderness (Hypothesis 1). Second, in line with Lishner et al. (2011), we aim to show that individuals score higher on the ERQ factor that reflects sympathy than on the ERQ factor that reflects tenderness in response to a need-situation that reflects current needs (Hypothesis 2).

Finally, for exploratory purposes, we examined potential gender differences in aspects of empathic concern. Previous studies have suggested that women are more empathic than men are (Eisenberg and Lennon 1983). The present research examined whether this gender difference regarding empathic concern could be replicated.

\section{Method}

Procedure and participants

Nine studies were conducted to examine the factor structure of that part of the ERQ that assesses empathic concern. The total sample consisted of 1,273 participants $(26.6 \%$ men, $73.4 \%$ women). Seven of the nine studies included both female and male participants, two studies (Studies 1 and 4) only female participants. In the seven studies with both male and female participants, the percentage of men ranged from $21 \%$ (Study 2) to $50 \%$ (Study 6). Participants were undergraduate students studying at the University of Groningen (Studies 1-7 and 9) and working individuals (Study 8). Participants were told that they were participating in a project on personality, interpersonal relationships and helping behaviour. Male and female experimenters were randomly assigned to assist participants and guide them through the research. Participants completed the study in separate cubicles. The average age across samples was 21.17 $(S D=2.88)$.

\section{Measures}

Empathy research often makes use of scenarios that intend to create a fictional but realistic need situation for participants and that induce feelings of empathic concern. One such scenario is the imaginary story of Katie Banks, a senior at university who recently lost her parents and a sister in a car accident. In this scenario, her parents did not have a life insurance, and, as a result, Katie is struggling to take care of her surviving younger brother and sister as well as finishing her last year of college. Following this story, the items of the ERQ are then often used to assess, among other emotions, empathic concern (see for instance Batson 1991; Batson et al. 1997; Coke et al. 1978; Mikulincer et al. 2001). In terms of the distinction made by Lishner et al. (2011), the Katie Banks scenario reflects a current need, and therefore should evoke sympathy, but not (necessarily) tenderness.

All of our nine studies used the same scenario, one that was based on the Katie Banks paradigm. The scenario describes the fictional story of Leonie, a female student who had a major bicycle accident and consequently ended up with serious facial damage, a shattered foot, and social stigma. In an interview, Leonie tells about her experiences in the hospital, how she felt when she saw herself again for the first time, how she has been recovering, and how she feels when people are staring at her. Similar to the Katie Banks scenario, this scenario primarily seems to reflect a current need. In Studies 1, 2 and 4, participants listened to an auditory interview with Leonie, in the other studies participants read the interview in the form of an article accompanied by a picture of Leonie. After listening to or reading the interview with Leonie, participants indicated, on 7 -point scales $(1=$ not at all, $7=$ very much $)$, the extent to which they experienced the six adjectives proposed by Batson et al. (1987), that is, sympathetic, moved, compassionate, tender, warm and soft-hearted. Table 1 shows the intercorrelations between these 6 adjectives averaged across the nine studies. It must be noted that three of our nine studies (Studies 1, 2 and 4) also included a perspective taking manipulation. For the present paper, the 
Table 1 Intercorrelations between the adjectives that assess empathic concern (averaged across studies)

All $p$ s $<.01$

three perspective taking conditions that were used in these studies were combined.

\section{Results}

\section{Factor analyses}

To test the expectation that empathic concern consists of two factors, that is, one reflecting sympathy and one reflecting tenderness, factor analyses (Principal Components Analyses) were first conducted extracting two factors, separately for each of the 9 datasets. In support of Hypothesis 1, Table 2 shows that in seven of the nine studies (i.e., Studies 1, 3, 4, 5, 6, 7, and 8) the two factor solution clearly distinguished between a 'sympathy cluster' and a 'tenderness cluster' of adjectives: whereas the adjectives compassionate, sympathetic, and moved had relatively high loadings on the first factor and relatively low loadings on the second factor, the opposite was found for the adjectives tender, warm, and softhearted. Some small anomalies were found: in Study 2, the adjective moved had its primary loading on the Tenderness factor, whereas in Study 9 the adjective tender had about equally high loadings on both factors. In both of these cases, the adjectives had high factor loadings on both factors.

In addition to the separate PCA's, we also conducted a Simultaneous Component Analysis (SCA; Kiers and Ten Berge 1994) extracting two factors. The purpose of this SCA is to find a single kernel structure that best reflects the factor structures in all (nine) different data sets. The SCA solution accounted for $68 \%$ of the variance, and, in support of Hypothesis 1, also revealed two clearly distinguishable factors across the nine studies, that is, a Tenderness factor and a Sympathy factor (see Table 2). Finally, because in the PCA solutions all six items had (in general) a substantial loading on the first unrotated principal component, we examined whether the reported two factor solution would fit the data better than a one factor solution. For this purpose, confirmatory analyses were conducted in the combined sample (using LISREL), testing a model with two factors (tenderness and sympathy), versus a model with one factor (empathy). It was found that the model with two factors (sympathy and tenderness) fit the data clearly better $(\mathrm{CFI}=.97)$ than the model with just a single empathy factor $(\mathrm{CFI}=.84)$.

For further analyses, on the basis of the abovementioned analyses, two scale scores were computed, one for Sympathy (summing the scores of the adjectives compassionate, sympathetic and moved) and one for Tenderness (summing the scores of the adjectives warm, tender and softhearted). When conducted separately for each data set, analyses showed that, for Studies 1-8, Cronbach's alphas for these scales ranged between .72 and .83 for the Sympathy scale and .68 and .89 for the Tenderness scale (see Table 3). In Study 9 both reliabilities were somewhat lower compared to the other studies (i.e., .66 and .63, respectively).

Sympathy and tenderness in response to the scenario

The mean scores for Sympathy and Tenderness in response to the scenario differed considerably across the nine studies: especially the differences in tenderness and sympathy between Study 8 and the other studies stands out (see Table 3). A possible explanation lies in Study 8's sample: whereas this sample consisted of working individuals, the samples of the other eight studies consisted of undergraduate students only. Probably related to this sample characteristic, analyses showed that participants in Study 8, on average, were somewhat older $(M=23$ years $)$ than participants in the other studies, which may explain, to some extent, their lower responses in terms of sympathy and tenderness, since age and both sympathy and tenderness were negatively related ( $r$ s across samples were -.19 and -.12 respectively, $p s<.001$ ). The correlations between the two subscales sympathy and tenderness varied between .22 and .55, indicating a small to moderate relation between the two subscales, providing further support for the idea that sympathy and tenderness are related, but different emotions. Confirming Hypothesis 2, participants responded with relatively strong sympathy (means ranging from 13.7 to 17.0 on a scale theoretically ranging from 3 to 21 ), but with neutral tenderness (means ranging from 8.8 to 13.5 on a scale theoretically ranging from 3 to 21 ) to the story of Leonie. Paired-sample $t$ tests showed that this 


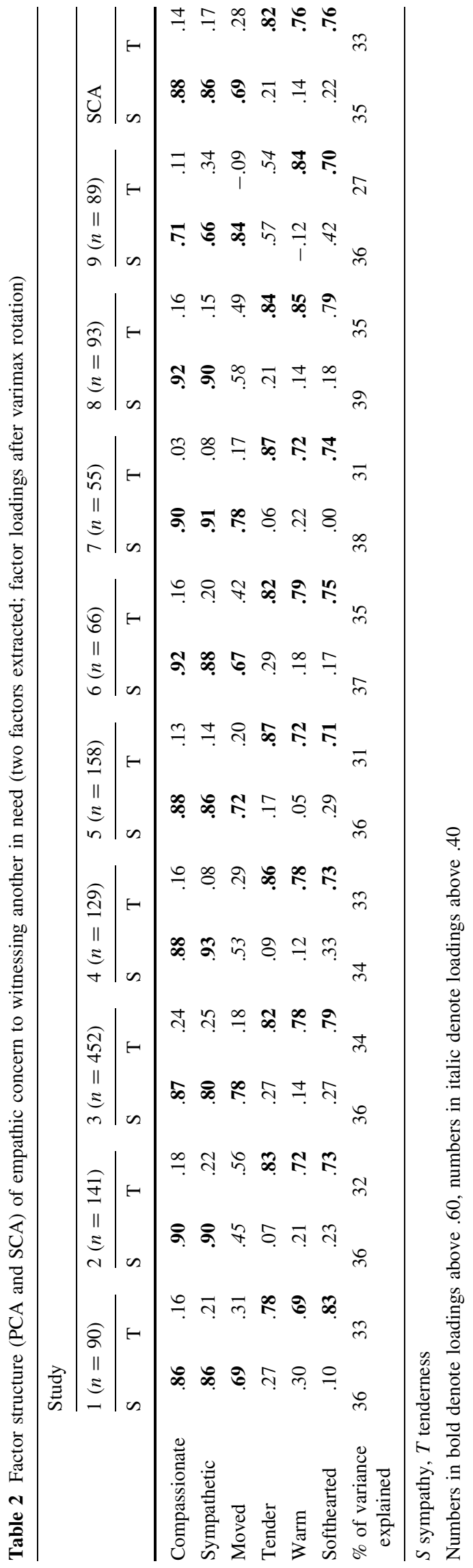

Table 3 Means ( $S D s$ ) and Cronbach's alphas for sympathy and tenderness and intercorrelations between sympathy and tenderness for each of the 9 studies

\begin{tabular}{|c|c|c|c|c|c|}
\hline \multirow[t]{2}{*}{ Study } & \multicolumn{2}{|c|}{ Sympathy } & \multicolumn{2}{|c|}{ Tenderness } & \multirow[t]{2}{*}{$r_{\mathrm{ST}}$} \\
\hline & $\alpha$ & $M(S D)$ & $\alpha$ & $M(S D)$ & \\
\hline $1(n=90)$ & .75 & $16.2(2.9)$ & .72 & $13.5(2.7)$ & .52 \\
\hline $2(n=141)$ & .72 & $16.2(2.8)$ & .71 & $12.5(3.2)$ & .55 \\
\hline $3(n=452)$ & .79 & $17.0(2.3)$ & .76 & $13.1(3.2)$ & .51 \\
\hline $4(n=129)$ & .70 & $15.4(3.0)$ & .74 & $10.8(3.6)$ & .40 \\
\hline $5(n=158)$ & .75 & $15.3(3.0)$ & .69 & $10.5(3.4)$ & .39 \\
\hline $6(n=66)$ & .82 & $15.0(3.2)$ & .75 & $9.5(3.5)$ & .54 \\
\hline $7(n=55)$ & .83 & $15.4(3.2)$ & .68 & $9.3(3.5)$ & .22 \\
\hline $8(n=93)$ & .80 & $13.7(3.6)$ & .81 & $8.8(3.8)$ & .51 \\
\hline $9(n=89)$ & .66 & $15.2(2.5)$ & .63 & $12.5(3.0)$ & .45 \\
\hline
\end{tabular}

$r_{\mathrm{ST}}$ refers to the correlation between sympathy and tenderness, $p \mathrm{~s}<.05$

difference was significant in all nine datasets $(t \mathrm{~s} \geq 8.71$, $p \mathrm{~s}<.001)$.

Gender differences in empathic concern

In five out of the seven studies that included both sexes we did not find a significant difference between men's and women's scores on sympathy or tenderness. In Studies 3 and 5 we found women to score significantly higher on sympathy than men [Study $3 M_{\text {women }}=17.3, S D_{\text {women }}=$ 2.1 vs. $M_{\text {men }}=16.3, S D_{\text {men }}=2.8, F(1,416)=15.76$, $p<.001$; Study $5 M_{\text {women }}=15.8, S D_{\text {women }}=3.1$ vs. $\left.M_{\text {men }}=14.8, S D_{\text {men }}=2.8, F(1,157)=5.05, p<.05\right]$. In not a single study men and women differed significantly in tenderness.

\section{Discussion}

The present research set out to show that the ERQ items that assess empathic concern can best be split into two subsets of items, that is, one reflecting sympathy and one reflecting tenderness. For this purpose nine studies were conducted in which participants were presented with a person in need, and asked to fill in the six adjectives of the ERQ that assess empathic concern. Analyses showed that, as expected, empathic concern can be split best into two components, that is, sympathy and tenderness. This finding fits well with recent findings by Lishner et al. (2011) showing that sympathy and tenderness are different empathy-related emotions. Also in line with Lishner et al. (2011) we found individuals to score higher on the ERQ factor that reflects sympathy than on the ERQ factor that reflects tenderness when confronted with a need-situation that reflects current needs. 
Our study did not support previous findings on gender differences in empathic concern. In only two of the seven studies women reported more feelings of sympathy than men. Regarding tenderness no gender differences were found at all. A possible explanation is that The Netherlands is a relatively 'feminine' country in terms of Hofstede's theory on cultural dimensions (1980). In feminine cultures gender roles show high overlap, and both men and women are to some extent modest, tender, and concerned with the quality of life. As a consequence, it can be expected that Dutch men and women respond more alike in terms of tenderness and sympathy than men and women from cultures that are more masculine, such as the US.

\section{Implications and future research}

Our finding that empathic concern consists of two emotions, one reflecting sympathy and one tenderness, has several important implications. Most importantly, the present research helps better understand the nature of empathic concern. For long scholars have argued about the proper definition of empathy (usually equating empathy with empathic concern, and not other forms of empathy, such as empathic anger), and both operational definitions and theoretical definitions of empathy vary widely across studies and show considerable inconsistencies. For instance, Titchener (1909), who first coined the term empathy, considered empathy either as the subject's awareness in imagination of the emotions of another person as well as a kind of social-cognitive bonding. Within a clinical context, empathy was initially viewed as a cognitive process referring to accurately and dispassionately understanding the client's point of view concerning his or her situation (Dymond 1949). Rogers (1951, p. 29) referred to empathy as the focus of the therapist when trying to "live the attitudes of the other". Eventually, definitions of empathy, and with it empathy measures, shifted from cognition-based to emotion-based (Coke et al. 1978; Stotland 1969; Mehrabian and Epstein 1972). That is, empathy was increasingly viewed as a vicarious emotion, that is, feeling either the same emotion as the other person, or an emotion congruent with (but not necessarily identical to) the emotion of the other person (Batson and Coke 1981; Eisenberg and Strayer 1987; Stotland 1969). The distinction between tenderness and sympathy may help understand the discrepancies between these different definitions. For instance, more than in other contexts, in the clinical context, definitions of empathy may have been driven relatively strongly by current needs with which clients struggle, and, consequently, emphasize the experience of sympathy.

The distinction between sympathy and tenderness may also help locate the specific roots of helping behavior. In addition to resulting from different needs (Lishner et al. 2011), tenderness and sympathy may also have different motivational consequences for subsequent helping intentions. Because sympathy, but not tenderness, is evoked by current needs (Lishner et al. 2011), it seems most likely that sympathy rather than tenderness motivates actual helping behavior. This possibility cast a new light on previous studies that based their results on the total score of the six items assessing empathic concern. Several studies have, for instance, shown that empathically concerned individuals (as indicated by the total score on the six empathy items of the ERQ) help those in need, even when physical or psychological escape from the need situation is easy (e.g., Batson and Coke 1981; Stocks et al. 2009). From the perspective of the present research, these findings raise questions, such as: Do both aspects of empathic concern direct altruistic behaviors, regardless of opportunities to psychologically or physically escape? Or is it the component of sympathy only that motivates helping behavior even under conditions of escape? What happens with the component of tenderness when individuals are provided with the opportunity to escape a need-situation? It is important for future research to examine these issues. If, indeed, sympathy is the single motivating component of altruism, this may further enhance our understanding of helping behaviors, the emotions that give rise to it, and the conditions under which individuals help others in need. Finally, our study suggests that the component of empathic concern that gives rise to altruistic helping behavior may be assessed more effectively, efficiently and reliably by means of the three items that our study found to reflect sympathy, rather than by the six original empathy items of the ERQ.

Of course our research also has its limitations. First, the present research relied on imagined hypothetical scenarios that may have not been perceived as realistic to all participants. Second, the use of a specific scenario may limit the generalizability of findings to other situations of need. Third, although we assumed the scenario to reflect current needs (in contrast to vulnerability), we did not assess the extent to which participants indeed perceived this to be the case. Finally, mean differences in tenderness and sympathy may merely reflect differences in intensity, rather than basic conceptual differences. Despite these limitations, we believe the present research contributes significantly to the literature on empathy. By showing that tenderness and sympathy are different components of empathic concern, the present research emphasizes the importance of distinguishing between these emotions when examining emotional responses to persons in need. We hope our research inspires other scholars to distinguish between tenderness and sympathy and to study the different effects these different emotions may have on, for instance, the intention to help someone in need and actual helping behaviors. 
Open Access This article is distributed under the terms of the Creative Commons Attribution Noncommercial License which permits any noncommercial use, distribution, and reproduction in any medium, provided the original author(s) and source are credited.

\section{References}

Batson, C. D. (1991). The altruism question: Toward a socialpsychological answer. Hillsdale, NJ: Erlbaum.

Batson, C. D., \& Coke, J. S. (1981). Empathy: A source of altruistic motivation for helping? In J. P. Rushton \& R. M. Sorrentino (Eds.), Altruism and helping behaviour: Social, personality and developmental perspectives (pp. 167-187). Hillsdale, NJ: Lawrence Erlbaum Associates.

Batson, C. D., Cowles, C., \& Coke, J. S. (1979) Empathic mediation of the response to a lady in distress egoistic or altruistic? Unpublished manuscript. University of Kansas.

Batson, C. D., O'Quin, K., Fultz, J., Vanderplas, M., \& Isen, A. (1983). Influence of self-reported distress and empathy on egoistic versus altruistic motivation to help. Journal of Personality and Social Psychology, 45, 706-718.

Batson, C. D., Fultz, J., \& Schoenrade, P. A. (1987). Distress and empathy: Two qualitatively distinct vicarious emotions with different motivational consequences. Journal of Personality, 55, 19-39.

Batson, C. D., Early, S., \& Salvarani, G. (1997). Perspective taking: Imagining how another feels versus imagining how you would feel. Personality and Social Psychology Bulletin, 23, 751-758.

Coke, J. S. (1980). Empathic mediation of helping egoistic or altruistic? (Doctoral dissertation, University of Kansas, 1979) Dissertation Abstracts International, $41 B$ (01), 405 (University Microfilms no. 8014371).

Coke, J. S., Batson, C. D., \& McDavis, K. (1978). Empathic mediation of helping: A two-stage model. Journal of Personality and Social Psychology, 36, 752-766.

Dymond, R. (1949). A scale for the measurement of empathy ability. Journal of Consulting Psychology, 13, 127-133.

Eisenberg, N., \& Lennon, R. (1983). Sex differences in empathy and related capacities. Psychological Bulletin, 94, 100-131.

Eisenberg, N., \& Strayer, J. (1987). Critical issues in the study of empathy. In N. Eisenberg \& J. Strayer (Eds.), Empathy and its development (pp. 3-13). Cambridge: Cambridge University Press.

Fultz, J. N. (1982). Influence of potential for self-reward on egoistically and altruistically motivated helping. Unpublished Master's thesis, University of Kansas.

Hofstede, G. (1980). Culture's consequences: International differences in work-related values. Beverly Hills, CA: Sage.
Kalawski, J. P. (2010). Is tenderness a basic emotion? Motivation and Emotion, 34, 158-167.

Kiers, H. A. L., \& Ten Berge, J. M. F. (1994). Hierarchical relations between methods for simultaneous component analysis and a technique for rotation to a simple simultaneous structure. British Journal of Mathematical and Statistical Psychology, 47, $109-126$.

Lishner, D.A. (2003). The components of empathy: Distinguishing between tenderness and sympathy. Unpublished doctoral dissertation, University of Kansas, Lawrence.

Lishner, D. A., Batson, D. C., \& Huss, E. (2011). Tenderness and sympathy: Distinct empathic emotions elicited by different forms of need. Personality and Social Psychology Bulletin, 37, 614-625.

McDougall, W. (1908). An introduction to social psychology (18th ed.). London: Methuen.

Mehrabian, A., \& Epstein, N. (1972). A measure of emotional empathy. Journal of Personality, 40, 525-533.

Mikulincer, M., Gillath, O., Halevy, V., Avihou, N., Avidan, S., \& Eshkoli, N. (2001). Attachment theory and reactions to others' needs: Evidence that activation of the sense of attachment security promotes empathic responses. Journal of Personality and Social Psychology, 81, 1205-1224.

Rogers, C. (1951). Client-centered therapy. Boston: HoughtonMifflin.

Shaver, P., Schwartz, J., Kirson, D., \& O'Connor, C. (1987). Emotion knowledge: Further exploration of a prototype approach. Journal of Personality and Social Psychology, 52, 1061-1086.

Stocks, E., Lishner, D. A., \& Decker, S. K. (2009). Altruism or psychological escape: Why does empathy promote prosocial behavior? European Journal of Social Psychology, 39(5), 649-665.

Stocks, E. L., Lishner, D. A., Waits, B. L., \& Downum, E. M. (2011). I'm embarrassed for you: The effect of valuing and perspective taking on empathic embarrassment and empathic concern. Journal of Applied Social Psychology, 41, 1-26.

Stotland, E. (1969). Exploratory investigations of empathy. In L. Berkowitz (Ed.), Advances in experimental social psychology (Vol. 4, pp. 271-313). New York: Academic Press.

Titchener, E. (1909). Elementary psychology of the thought processes. New York: Macmillan.

Toi, M., \& Batson, C. D. (1982). More evidence that empathy is a source of altruistic motivation. Journal of Personality and Social Psychology, 43, 281-292.

Woltin, K. A., Corneille, O., Yzerbyt, V. Y., \& Förster, J. (2011). Narrowing down to open up for other people's concerns: Empathic concern can be enhanced by inducing detailed processing. Journal of Experimental Social Psychology, 47, $418-424$. 